\title{
Osteoarthritis: New Insights in Animal Models
}

\author{
Umile Giuseppe Longo ${ }^{*}, 1,2$, Mattia Loppini ${ }^{1,2}$, Caterina Fumo ${ }^{1,2}$, Giacomo Rizzello ${ }^{1,2}$, \\ Wasim Sardar Khan ${ }^{3}$, Nicola Maffulli ${ }^{4}$ and Vincenzo Denaro ${ }^{1,2}$
}

\author{
${ }^{I}$ Department of Orthopaedic and Trauma Surgery. Campus Bio-Medico University, Via Alvaro del Portillo, 200, 00128 \\ Trigoria, Rome, Italy \\ ${ }^{2}$ Centro Integrato di Ricerca (CIR) Campus Bio-Medico University, Via Alvaro del Portillo, 21, 00128, Rome, Italy \\ ${ }^{3}$ University College London Institute of Orthopaedics and Musculoskeletal Sciences, Royal National Orthopaedic \\ Hospital, Stanmore, London, HA7 4LP, UK \\ ${ }^{4}$ Centre for Sports and Exercise Medicine, Barts and The London School of Medicine and Dentistry, Mile End Hospital, \\ 275 Bancroft Road, London, E1 4DG, UK
}

\begin{abstract}
Osteoarthritis (OA) is the most frequent and symptomatic health problem in the middle-aged and elderly population, with over one-half of all people over the age of 65 showing radiographic changes in painful knees. The aim of the present study was to perform an overview on the available animal models used in the research field on the OA. Discrepancies between the animal models and the human disease are present. As regards human 'idiopathic' OA, with late onset and slow progression, it is perhaps wise not to be overly enthusiastic about animal models that show severe chondrodysplasia and very early OA. Advantage by using genetically engineered mouse models, in comparison with other surgically induced models, is that molecular etiology is known. Find potential molecular markers for the onset of the disease and pay attention to the role of gender and environmental factors should be very helpful in the study of mice that acquire premature OA. Surgically induced destabilization of joint is the most widely used induction method. These models allow the temporal control of disease induction and follow predictable progression of the disease. In animals, ACL transection and meniscectomy show a speed of onset and severity of disease higher than in humans after same injury.
\end{abstract}

Keywords: Osteoarthritis, animal models, genetic engineering, molecular markers.

\section{INTRODUCTION}

Osteoarthritis (OA) is the most frequent and symptomatic health problem in the middle-aged and elderly population, with over one-half of all people over the age of 65 showing radiographic changes in painful knees [1]. It is characterized by join pain, stiffness, hypertrophy and limitation in the joint range of motion. OA affects not only the articular cartilage, but the whole joint including ligaments, muscles, subchondral bone, menisci (in the knee), synovium, capsule and joint fluid. Instability and inflammation within the joint leads to changes in several cells, such as osteoblasts that proliferate along the joint margin leading to the osteophyte formation [2], synovial cells that proliferate causing hyperplasia of the synovium [3] and subchondral bone formation leading to sclerosis underlying damaged cartilage [4].

Primary OA progresses slowly and is the most common type of OA. Post-traumatic OA develop more rapidly, and it can be produced by mechanical insults or surgical lesions

*Address correspondence to this author at the Department of Orthopaedic and Trauma Surgery, Campus Bio-Medico University, Via Alvaro del Portillo, 200, 00128 Trigoria, Rome, Italy; Centro Integrato di Ricerca (CIR) Campus Bio-Medico University, Via Alvaro del Portillo, 21, 00128, Rome, Italy; Tel.: + 39062254 11; Fax: + 3906 225411934;

E-mail: g.longo@unicampus.it leading to joint instability. Etiology, pathogenesis and progression of $\mathrm{OA}$ are not completely understood yet. Recent studies underlined the central role for inflammatory cytokines as biochemical signals, that boost chondrocytes to release cartilage-degrading proteinases. An animal model for human disease can be defined as a homogeneous set of animals which have an inherited, naturally acquired, or experimentally induced biological process, amenable to scientific investigation, that in one or more respects resembles the disease in humans [5].

Several animal models have been described for the study of osteoarthritis, however the gold standard animal model is not be found yet. It is difficult to standardize across clinical units [6]. In terms of standard histologic changes there is no consensus on the common grading systems. The Mankin scoring system is useful, but has some limitations particularly in early OA. Biochemical biomarkers are useful to predict OA progression, especially the condition of cartilage, bone turnover and synovitis. Finally, it should identify the disease activity prior to radiographic change are detected by MRI or other radiological methods [7].

The correct and ethical use of animals in the research field should follow the "3Rs" approach: Reduce the number of animal used; Refine the procedures and protocols to minimize suffering and maximize the value of the outcomes; and where possible Replace animal studies with an 
alternative approach [6]. The Arrive (Animal in Research: Reporting In Vivo Experiments) guidelines can drive the researcher to choose and evaluate the limitations of each animal model. Arrive guidelines consists of 20 items describing the minimum information that all scientific publications reportage research using animals should include, such as the number and specific characteristics of animal used (includes species, strain, sex, and genetic background); details of housing and husbandry and the experimental, statistical and analytical methods.

The aim of the present study was to perform an overview on the available animal models used in the research field on the $\mathrm{OA}$.

\section{SURGICAL INDUCED OA MODELS}

\section{Temporomandibular Joint}

L. Xu et al. developed a mouse model of OA performing a partial discectomy in the temporomandibular joint (TMJ). In the control group, mice underwent similar preparation and surgical procedure but their discs were not cut. Body weights of all the mice were recorded prior to surgery and three times per week after the surgery. At 2, 4, 8 and 16 weeks postsurgery, eight mouse's heads from the discectomy group and eight mouse's heads from the sham-surgery group were fixed in $4 \%$ paraformaldehyde for 6 hours at room temperature. The morphology of articular cartilages was evaluated using a modified Mankin score system. The expression of discoidin domain receptor 2, matrix metalloproteinase-13 (MMP-13), and MMP-13 derived type II collagen fragments in the articular cartilage of condyles from the mouse TMJ were examined through immunohistostaining. There were no significant variations in body weights of the experimental and control mice. Partial discectomy consisted in removal of a half to two thirds of the disc, but there was no correlation between size of removed disc and severity of the articular cartilage degeneration. On the other hands, the severity of the cartilage damage was associated with the time course following the surgery. At 4 weeks after surgery proteoglycan staining in the extracellular matrix increases, at 8 weeks chondrocyte clusters appear, at 12 weeks there are fibrillation and a reduction of proteoglycan staining, and at 16 weeks articular cartilage decreases. This early onset OA should be due to changes in distribution of the pressure and in the capacity of load absorption on articular surfaces of the TMJ following the partial removal of the disc. This results in an excessive mechanical force acting on a small area of articular surface with subsequent early-onset OA [8].

\section{Anterior Cruciate Ligament Transection (ACLT) and/or Meniscectomy}

\section{Rat Model}

Sequential events of early cartilage degradation, subchondral osteopenia followed by sclerosis, and late osteophyte formation were demonstrated in the anterior cruciate ligament transaction (ACLT) or ACLT with partial medial meniscectomy (ACLT+MMx) or partial medial meniscectomy rat OA models $[9,10]$. Changes in gene expression during OA progression were demonstrated by using known markers. 10 week old-male rat were surgically treated to induce OA in right knee joint. The knee joint was exposed with the medial parapatellar approach. The patella was dislocated laterally and the knee placed in full flexion. The anterior cruciate ligament (ACL) was then transected with micro-scissors. In the more severe model of OA, ACLT with partial medial meniscectomy, the medial collateral ligament was additionally transected with resection of medial meniscus. The time points of the study were at 1,2 , 4, 6 and 10 weeks after surgery [9]. At each time points, three cuts from the end of each tibia were performed. In slice one, approximately $960 \mu \mathrm{m}$ from the tibial plateau, articular cartilage and subchondral bone are inclused. Slice two included epiphyseal bone and growth plate, whereas slice three consisted of metaphyseal bone. The authors performed a comparison of gene expression of aggrecanase- 1 and MMP-13 between ACLT and ACLT+MMx models versus control animals, in which a sham procedure was performed, with PCR and immunohistochemical methods. Aggrecanase1 and MMP-13 were up-regulated in both OA models compared to sham procedure group. Expression levels of aggrecanase-1 increased 3-4 fold in both ACLT+MMx and ACLT models compared to controls. The relevant role of aggrecanase- 1 in cartilage degeneration during the initial and progressive phases of OA is highlighted by elevated mRNA expression levels above controls up to 10 weeks after surgery. The regulation of MMP-13 synthesis was similar to that of aggrecanase-1 in ACLT and ACLT+MMx models.

\section{Canine Model}

Canine models offer advantages over rodent models because the complement of MMPs and degradation products are more consistent with humans [10] and the growth plates in dogs close with maturity, unlike those in rats. Joint laxity in dogs is followed by OA and spontaneous rapture of the anterior cruciate ligament (ACL) [11]. In a canine model, 8 weeks after ACLT, osteophytes and cartilage erosions on both condyles and plateau were detected [11]. These changes are associated with enhanced production of two major protease involved in bone resorption, such as cathepsin $\mathrm{K}$ and MMP-13 [12]. Studies demonstrated an important role of TGF- $\beta$ to induce the synthesis of MMP-13 [13]. Movement of TGF- $\beta$ from the subchondral bone region to the basal layer of cartilage in OA process, appears favored by the presence of many channels between the subchondral region and uncalcified cartilage [14] and by the presence of microcracks in the articular cartilage [15]. Cartilage biomarkers (aggrecan 846 epitope for intact aggrecan proteoglycan and Col2-3/C4C $\mathrm{C}_{\text {long mono }}$ and Col2CTx for type II collagen degradation) were elevated in joint fluid obtained from dogs soon after anterior cruciate transaction [16]. Joint degeneration and features of early OA caused by damage to the weight-bearing areas of the articular cartilage in the femoral condyles of the knee, have been observed in the "Groove" canine model $[17,18]$. To enhance the effect of this trigger, the loading on the affected joint is increased by fixing the contra-lateral health limb to the trunk of the dog intermittently ( 2 hours for 3 days a week). The results obtained are similar to biochemical and histological evaluations of degenerative changes in the joint in the anterior cruciate ligament transection (ACLT) model. In the canine Groove model at 10 weeks, collagen is damaged and proteoglycan (PG) turnover is impaired; an ineffective synthesis combined with an enhanced release results in diminished PG content. Matrix metalloproteinase (MMP) 
activity in synovial fluid is enhanced. Loss of safranin-O staining, fibrillation of the articular surface and chondrocyte clustering indicate moderate cartilage destruction. These characteristics of $\mathrm{OA}$ are observed in the tibial plateau, although this cartilage surface is not harmed during surgery. Only mild signs of inflammation are present. Degenerative changes in the cartilage matrix integrity progress slowly in the first year after surgery, while synovial inflammation decreases.

\section{Rabbit Model}

ACLT is used in rabbit models to induce osteoarthritis. The age at which joint maturity is attained will depend on the joint. A recent study reported that rabbit femorotibial joint articular cartilage matures much earlier at approximately 3 months [19]. On the other hands, older adult animals would be preferable models of human OA, but it is very difficult to obtain large number of adult animals for studies [20]. About the timeframe of development of the disease, fissures and erosion of cartilage can occur as early as 4 weeks post-ACLT. The cartilage lesions observed in each compartment are focal and usually located in the femoral condyles and in the tibial plateau. Inter-animal variation in lesion's development is encountered, probably related to the use of different batch of animals with a different genetic background. Moreover, speed of onset and severity of disease is higher than in humans after the same injury. Osteophytes always occur in femoral condyles and tibial plateau. Osteophytes indicated hypertrophic chondrocytes expressing VEGF. VEGF should favorite cartilage vascular invasion [21]. In rabbit ACLT model is still observed VEGF expression after the $4^{\text {th }}$ week [21]. VEGF stimulates OA chondrocytes to produce increased amounts of MMP-1 and MMP-3. VEGF has recently been shown to be expressed in hypertrophic cartilage in the growth plate and to coordinate hypertrophy, vascular invasion and angiogenesis [22]. Rabbit partial meniscectomy model of OA showed increased levels of IL-1 and IL-1 receptors, which seem related to the upregolation of prostromelysin, procollagenase, and cathepsin B gene expression in the degenerated cartilage $[23,24]$. IL- 1 is the pivotal cytokine at early and late stages, while TNF- $\alpha$ is involved in the onset of arthritis [25]. The single injection of highly purified or recombinant IL-1 into knee joints of rabbits induces a marked cellular infiltrate in synovial lining and fluid, and the loss of proteoglycan from the articular cartilage $[26,27]$. A new video imaging technique to quantify and localize Indian ink staining of cartilage has showed that ACLT- induced degeneration had a predilection for the posteromedial aspects of the joint, and to a lesser extent, the anterolateral aspects [28].

\section{Ovine Model}

In ovine meniscectomy model, typical degenerative changes are observed in the operated knee joints at three months after surgery. Proteoglycan 4 is a lubricating glycoprotein considered to be primarily responsible for border lubrication in synovial joints. In the early phases of OA, changes in expression and localization of proteoglycan 4 occurred. In normal control joints, proteoglycan 4 was immunolocalized in the superficial zone of cartilage, particularly in those regions of the knee joint covered by a meniscus. After the onset of early osteoarthritis, a loss of cellular proteoglycan 4 immunostaining in degenerative articular cartilage, was observed [29].

\section{ENZYMATICALLY/CHEMICALLY INDUCED OA MODELS}

Chemical models include intra-articular injection of substances having various effects on joint physiology such as inhibition of chondrocyte metabolism, including papain or iodoacetate, damage of ligaments and tendons, including collagenase, or selective joint denervation, including immunotoxins [30].

In rat models, iodoacetate injection is used to induce OA damage [6]. Joint degeneration induced by iodoacetate injection is chronic and depends on the concentration of iodoacetate: 29 days after the injection of $0.3 \mathrm{mg}$ of iodoacetate, no joint destruction was visible; while relievable joint degeneration was present after 1 or $3 \mathrm{mg}$ of iodoacetate injection [31]. Osteolysis and swelling with displacement of the patella characterized joint degeneration in rat's knee [3133]. Joint pathology was caused by the inhibition of glycolysis, thereby targeting the avascular cartilage and causing chondrocyte death, fragmentation of cartilage, and exposure of subchondral bone [10,34]. This model has some limitation: iodoacetate is a metabolic poison, and chondrocyte death is wide, differently from human OA [7]. On the other hands, the intra-articular injection of papain does not conduct to chronic alterations in joint integrity [31].

Because of the presence of fibronectin fragments in synovial fluid of patients with OA [35], fibronectin fragments were injected in rabbit knee and causes a depletion of cartilage proteoglycans [36]. Chondrolysis is associated with relapsing of stromelysin, IL-1 and other catabolic interleuchin, caused by fibronectin fragments [37, 38].

\section{SPONTANEOUS MODELS}

In about $38-65 \%$ of patients with OA the disease results from the genetic background [39]. OA onset appears connected to mutations of only a few of human genes, especially those coding for cartilage-specific collagens. It seems that any alteration in a structural gene encoding a cartilage matrix constituent can surround defect of normal properties of the tissue and lead to early onset OA [40] and other bone and cartilage lesions (i.e. condrodysplasias) [41].

Guinea pigs develop spontaneous OA in knee and other joints. Since 3 months of age, histologic changes occur, and at 18 months moderate to severe OA is observed in the medial tibial plateau cartilage which is not covered by menisci. This is due partly to obesity and to various positioning of the hind limbs [42, 43]. Spontaneous NO release from the knee cartilage of the guinea pig increases with age and the OA development is observed, with a decrease of intracellular ATP levels in chondrocytes and a progressive elevation of chondrocyte ATP-scavenging nucleotide pyrophosphatase/phosphodiesterase (NPP) activity and extracellular levels of the NPP enzymatic end product inorganic pyrophosphate (PPi). These findings suggest a role of NO in mitochondrial dysfunction and cartilage calcification in OA [44]. 
STR/ort mice are a model for spontaneous OA with a tendency for patella displacement. They exhibit elevated levels for both local and systemic inflammatory markers. Spontaneous OA appears at approximately 12 to 20 weeks [45]. A high degree of degeneration in the medial side of the articular cartilage, subchondral sclerosis, and an increase in glycosaminoglycan content prior to the onset of degeneration are present in STR/ort mice. These features are similar to human OA. Moreover, this mice present elevated levels of both local and systemic inflammatory markers (RAGE, AGE, S100A4 and HMGB1) and cytokines (IL1 $\alpha$, IL1 $\beta$, IL6) [45]. OA phenotype does not follow Mendelian pattern of inheritance [46]. Biglycan and fibromodulin are small extracellular proteoglycans that are coexpressed in tendons, cartilage and bone. Mice with defects in non-collagenous molecules show tendon mineralization and OA (single knockout with fibromodulin deficiency) [47]. Biglycandeficient mice develop osteoporosis [48]. Double knockout, with fibromodulin and biglycan deficiency, show ectopic ossification and early onset $\mathrm{OA}$ in knee, like STR/ort mice [49]. Cre-Gdf5/Bmprla floxP mice are unable to produce a bone morphogenetic protein (BMP) receptor specifically in joints. These mice show retention of webbing between some digits, failure in formation of some joints in the ankles and premature OA in other joints, compared with normal wildtype mice [50]. In both byglican-fibromodulin double knockout mice $(B g n-F b n)$ and in Cre-Gdf5/Bmprla ${ }^{\text {floxP }}$, the OA onset was characterized by non-inflammatory fibrillation and subsequent thinning of the articular cartilage joint surfaces. Differences between this two models include selective joint fusion abnormalities and the limitation to the joint of the depletion of BMP signaling in Cre-Gdf5/Bmprla floxP, whereas in $B g n-F b n$ double knockout mice there is a total depletion of the two proteoglycans in all tissue and joint fusion abnormalities are not observed.

Other mouse models of OA present mutations or deletions of genes encoding several matrix and associated proteins. Transgenic mice whit defect in type II collagen show mild chondrodysplasia phenotype and OA [51, 52], transgenic mice whit defect I type IX collagens show OA and mild chondrodysplasia with ocular involvement [53]. Involvement of matrix metalloproteinases, especially MMP13 , has been demonstrated in the degradation of cartilage and the development of human OA lesions [54]. Transgenic mice over expressing MMP-13 in articular cartilage showed cartilage changes similar to human OA [55], while MMp-13 knockout mice displayed a reduction of tibial cartilage erosions in a surgical model of OA [56]. Enhanced expression of MMP-13 is related to an increased expression of the collagen receptor discoidin domain receptor (DDR-2) in transgenic mice that show OA cartilage pathology at 6 months of age $[57,58]$. ADAMTS-5 is the major aggrecanase involved in early proteoglycan loss in arthritis and OA. Transgenic aggrecan knocking mice had a mutation that made the interglobular domain (IGD) resistant to aggrecanase. This feature allowed for abrogate cartilage erosion in experimental OA and also for repair of depleted tissue [59].

\section{CONCLUSION}

Development of new animal models of OA is strongly related to enhanced research activity, but at the present the gold standard OA animal model has not been found yet. First of all because the pathology of human OA is not completely understood in all tissues. Therefore, replication in an animal model is not easy. There are no certainties whether OA etiology should be a cartilage- driven or a bone-driven process. Moreover, it is not clear if each animal model reproduces only a subset of cases because of the heterogeneity of human OA. Secondly, the time frame of disease's progression in animal models should be suitable to obtain appropriate valuations about early, mid and late pathophysiology and treatments effects. However, the time of progression of the disease in human is extremely variable, and generally longer for both development and progression. From this point of view, spontaneous models are more like human because of disease's slower onset and progression [7]. Thirdly, the animal should be a mammalian species that is tractable, inexpensive, easy to house and manage, large enough to allow multiple analyses/outcome measures (e.g. radiology, magnetic resonance imaging, synovial fluid analysis etc.). Finally, the animal models should be predictive of therapeutic disease modification in humans: "what works in the animal, works in patients". Although the genetic constitution of laboratory animals is basically similar to that of humans, with great similarity in gene structure and the tissue distribution of gene products, differences from humans arise with respect to body size and stature in small mammals walking on four feet. Moreover, the distribution of loads across the joints is quite different from that in humans. Each species has characteristic biological properties of the articular cartilage, i.e. adult human articular cartilage has a cell density one tenth of that in adult rabbits and one fifth of that in dogs. Therefore, discrepancies between the animal models and the human disease are present. As regards human 'idiopathic' OA, with late onset and slow progression, it is perhaps wise not to be overly enthusiastic about animal models that show severe chondrodysplasia and very early OA [48]. Advantage by using genetically engineered mouse models, in comparison with other surgically induced models, is that molecular etiology is known. Find potential molecular markers for the onset of the disease and pay attention to the role of gender and environmental factors should be very helpful in the study of mice that acquire premature OA [50]. Surgically induced destabilization of joint is the most widely used induction method. These models allow the temporal control of disease induction and follow predictable progression of the disease. In animals, ACLT and meniscectomy show a speed of onset and severity of disease higher than in humans after same injury.

\section{CONFLICT OF INTEREST}

The authors confirm that this article content has no conflict of interest.

\section{ACKNOWLEDGEMENTS}

Declared none.

\section{REFERENCES}

[1] Hinton R, Moody RL, Davis AW, Thomas SF. Osteoarthritis: diagnosis and therapeutic considerations. Am Fam Phys 2002; 65: 841-8.

[2] Mankin HJ, Lippiello L. Biochemical and metabolic abnormalities in articular cartilage from osteo-arthritic human hips. J Bone Joint Surg Am 1970; 52: 424-34. 
[3] Lindblad S, Hedfors E. Arthroscopic and immunohistologic characterization of knee joint synovitis in osteoarthritis. Arthritis Rheum 1987; 30: 1081-8.

[4] Bendele AM, Hulman JF. Spontaneous cartilage degeneration in guinea pigs. Arthritis Rheum 1988; 31: 561-5.

[5] Pritzker KP. Animal models for osteoarthritis: processes, problems and prospects. Ann Rheum Dis 1994; 53: 406-20.

[6] Little CB, Zaki S. What constitutes an "animal model of osteoarthritis"--the need for consensus? Osteoarthritis Cartilage 2012; 20: 261-7.

[7] Poole R, Blake S, Buschmann M, et al. Recommendations for the use of preclinical models in the study and treatment of osteoarthritis. Osteoarthritis Cartilage 2010; 18 (Suppl 3): S10-16.

[8] $\mathrm{Xu} \mathrm{L,} \mathrm{Polur} \mathrm{I,} \mathrm{Lim} \mathrm{C,} \mathrm{et} \mathrm{al.} \mathrm{Early-onset} \mathrm{osteoarthritis} \mathrm{of} \mathrm{mouse}$ temporomandibular joint induced by partial discectomy. Osteoarthritis Cartilage 2009; 17: 917-22.

[9] Pickarski M, Hayami T, Zhuo Y, Duong le T. Molecular changes in articular cartilage and subchondral bone in the rat anterior cruciate ligament transection and meniscectomized models of osteoarthritis. BMC Musculoskelet Disord 2011; 12: 197.

[10] Fernihough J, Gentry C, Malcangio M, et al. Pain related behaviour in two models of osteoarthritis in the rat knee. Pain 2004; 112: 8393.

[11] Jovanovic DV, Fernandes JC, Martel-Pelletier J, et al. In vivo dual inhibition of cyclooxygenase and lipoxygenase by ML-3000 reduces the progression of experimental osteoarthritis: suppression of collagenase 1 and interleukin-1beta synthesis. Arthritis Rheum 2001; 44: 2320-30.

[12] Pelletier JP, Boileau C, Brunet J, et al. The inhibition of subchondral bone resorption in the early phase of experimental dog osteoarthritis by licofelone is associated with a reduction in the synthesis of MMP-13 and cathepsin K. Bone 2004; 34: 527-38.

[13] Moldovan F, Pelletier JP, Hambor J, Cloutier JM, Martel-Pelletier J. Collagenase-3 (matrix metalloprotease 13) is preferentially localized in the deep layer of human arthritic cartilage in situ: in vitro mimicking effect by transforming growth factor beta. Arthritis Rheum 1997; 40: 1653-61.

[14] Clark JM. The structure of vascular channels in the subchondral plate. J Anat 1990; 171: 105-15.

[15] Sokoloff L. Microcracks in the calcified layer of articular cartilage. Arch Pathol Lab Med 1993; 117: 191-5.

[16] Matyas JR, Atley L, Ionescu M, Eyre DR, Poole AR. Analysis of cartilage biomarkers in the early phases of canine experimental osteoarthritis. Arthritis Rheum 2004; 50: 543-52.

[17] Marijnissen AC, van Roermund PM, Verzijl N, Tekoppele JM, Bijlsma JW, Lafeber FP. Steady progression of osteoarthritic features in the canine groove model. Osteoarthritis Cartilage 2002; 10: 282-9.

[18] Marijnissen AC, van Roermund PM, TeKoppele JM, Bijlsma JW, Lafeber FP. The canine 'groove' model, compared with the ACLT model of osteoarthritis. Osteoarthritis Cartilage 2002; 10: 145-55.

[19] Hunziker EB, Kapfinger E, Geiss J. The structural architecture of adult mammalian articular cartilage evolves by a synchronized process of tissue resorption and neoformation during postnatal development. Osteoarthritis Cartilage 2007; 15: 403-13.

[20] Laverty S, Girard CA, Williams JM, Hunziker EB, Pritzker KP. The OARSI histopathology initiative - recommendations for histological assessments of osteoarthritis in the rabbit. Osteoarthritis Cartilage 2010; 18 (Suppl 3): S53-65.

[21] Hashimoto S, Creighton-Achermann L, Takahashi K, Amiel D, Coutts RD, Lotz M. Development and regulation of osteophyte formation during experimental osteoarthritis. Osteoarthritis Cartilage 2002; 10: 180-7.

[22] Gerber HP, Vu TH, Ryan AM, Kowalski J, Werb Z, Ferrara N. VEGF couples hypertrophic cartilage remodeling, ossification and angiogenesis during endochondral bone formation. Nat Med 1999; 5: 623-8.

[23] Mehraban F, Kuo SY, Riera H, Chang C, Moskowitz RW. Prostromelysin and procollagenase genes are differentially upregulated in chondrocytes from the knees of rabbits with experimental osteoarthritis. Arthritis Rheum 1994; 37: 1189-97.

[24] Mehraban F, Tindal MH, Proffitt MM, Moskowitz RW. Temporal pattern of cysteine endopeptidase (cathepsin B) expression in cartilage and synovium from rabbit knees with experimental osteoarthritis: gene expression in chondrocytes in response to interleukin-1 and matrix depletion. Ann Rheum Dis 1997; 56: 10815.

[25] Goldring MB. The role of cytokines as inflammatory mediators in osteoarthritis: lessons from animal models. Connect Tissue Res 1999; 40: 1-11.

[26] Pettipher ER, Higgs GA, Henderson B. Interleukin 1 induces leukocyte infiltration and cartilage proteoglycan degradation in the synovial joint. Proc Natl Acad Sci U S A 1986; 83: 8749-53.

[27] Henderson B, Pettipher ER. Arthritogenic actions of recombinant IL-1 and tumour necrosis factor alpha in the rabbit: evidence for synergistic interactions between cytokines in vivo. Clin Exp Immunol 1989; 75: 306-10.

[28] Chang DG, Iverson EP, Schinagl RM, et al. Quantitation and localization of cartilage degeneration following the induction of osteoarthritis in the rabbit knee. Osteoarthritis Cartilage 1997; 5: 357-72.

[29] Young AA, McLennan S, Smith MM, et al. Proteoglycan 4 downregulation in a sheep meniscectomy model of early osteoarthritis. Arthritis Res Ther 2006; 8: R41.

[30] Salo PT, Theriault E, Wiley RG. Selective ablation of rat knee joint innervation with injected immunotoxin: a potential new model for the study of neuropathic arthritis. J Orthop Res 1997; 15: 622-8.

[31] Pomonis JD, Boulet JM, Gottshall SL, et al. Development and pharmacological characterization of a rat model of osteoarthritis pain. Pain 2005; 114: 339-46.

[32] Nagase H, Kumakura S, Shimada K. Establishment of a novel objective and quantitative method to assess pain-related behavior in monosodium iodoacetate-induced osteoarthritis in rat knee. J Pharmacol Toxicol Methods 2012; 65: 29-36.

[33] Guzman RE, Evans MG, Bove S, Morenko B, Kilgore K. Monoiodoacetate-induced histologic changes in subchondral bone and articular cartilage of rat femorotibial joints: an animal model of osteoarthritis. Toxicol Pathol 2003; 31: 619-24.

[34] Janusz MJ, Hookfin EB, Heitmeyer SA, et al. Moderation of iodoacetate-induced experimental osteoarthritis in rats by matrix metalloproteinase inhibitors. Osteoarthritis Cartilage 2001; 9: 75160.

[35] Xie DL, Meyers R, Homandberg GA. Fibronectin fragments in osteoarthritic synovial fluid. J Rheumatol 1992; 19: 1448-52.

[36] Homandberg GA, Meyers R, Williams JM. Intraarticular injection of fibronectin fragments causes severe depletion of cartilage proteoglycans in vivo. J Rheumatol 1993; 20: 1378-82.

[37] Xie DL, Hui F, Meyers R, Homandberg GA. Cartilage chondrolysis by fibronectin fragments is associated with release of several proteinases: stromelysin plays a major role in chondrolysis. Arch Biochem Biophys 1994; 311: 205-12.

[38] Homandberg GA, Hui F, Wen C, et al. Fibronectin-fragmentinduced cartilage chondrolysis is associated with release of catabolic cytokines. Biochem J 1997; 321 ( Pt 3): 751-7.

[39] Spector TD, Cicuttini F, Baker J, Loughlin J, Hart D. Genetic influences on osteoarthritis in women: a twin study. BMJ 1996; 312: 940-3.

[40] Prockop DJ, Ala-Kokko L, McLain DA, Williams C. Can mutated genes cause common osteoarthritis? Br J Rheumatol 1997; 36: 8279.

[41] Prockop DJ, Kivirikko KI. Collagens: molecular biology, diseases, and potentials for therapy. Annu Rev Biochem 1995; 64: 403-34.

[42] Jimenez PA, Glasson SS, Trubetskoy OV, Haimes HB. Spontaneous osteoarthritis in Dunkin Hartley guinea pigs: histologic, radiologic, and biochemical changes. Lab Anim Sci 1997; 47: 598-601.

[43] Bendele AM. Animal models of osteoarthritis. J Musculoskelet Neuronal Interact 2001; 1:363-76.

[44] Johnson K, Svensson CI, Etten DV, et al. Mediation of spontaneous knee osteoarthritis by progressive chondrocyte ATP depletion in Hartley guinea pigs. Arthritis Rheum 2004; 50: 121625.

[45] Kyostio-Moore S, Nambiar B, Hutto E, et al. STR/ort mice, a model for spontaneous osteoarthritis, exhibit elevated levels of both local and systemic inflammatory markers. Comp Med 2011; 61: 346-55.

[46] Watanabe K, Oue Y, Miyamoto Y, Matsuura M, Mizuno Y, Ikegawa S. Identification of a quantitative trait locus for spontaneous osteoarthritis in STR/ort mice. J Orthop Res 2012; 30: 15-20. 
[47] Ameye L, Aria D, Jepsen K, Oldberg A, Xu T, Young MF. Abnormal collagen fibrils in tendons of biglycan/fibromodulindeficient mice lead to gait impairment, ectopic ossification, and osteoarthritis. FASEB J 2002; 16: 673-80.

[48] Helminen HJ, Saamanen AM, Salminen H, Hyttinen MM. Transgenic mouse models for studying the role of cartilage macromolecules in osteoarthritis. Rheumatology (Oxford) 2002; 41: 848-56.

[49] Mason RM, Chambers MG, Flannelly J, Gaffen JD, Dudhia J, Bayliss MT. The STR/ort mouse and its use as a model of osteoarthritis. Osteoarthritis Cartilage 2001; 9: 85-91.

[50] Young MF. Mouse models of osteoarthritis provide new research tools. Trends Pharmacol Sci 2005; 26: 333-5.

[51] Helminen HJ, Kiraly K, Pelttari A, et al. An inbred line of transgenic mice expressing an internally deleted gene for type II procollagen (COL2A1). Young mice have a variable phenotype of a chondrodysplasia and older mice have osteoarthritic changes in joints. J Clin Invest 1993; 92: 582-95.

[52] Vandenberg P, Khillan JS, Prockop DJ, Helminen H, Kontusaari S, Ala-Kokko L. Expression of a partially deleted gene of human type II procollagen (COL2A1) in transgenic mice produces a chondrodysplasia. Proc Natl Acad Sci U S A 1991; 88: 7640-4.

[53] Nakata K, Ono K, Miyazaki J, et al. Osteoarthritis associated with mild chondrodysplasia in transgenic mice expressing alpha 1(IX) collagen chains with a central deletion. Proc Natl Acad Sci U S A 1993; 90: 2870-4.

[54] Shlopov BV, Lie WR, Mainardi CL, Cole AA, Chubinskaya S, Hasty KA. Osteoarthritic lesions: involvement of three different collagenases. Arthritis Rheum 1997; 40: 2065-74.

[55] Neuhold LA, Killar L, Zhao W, et al. Postnatal expression in hyaline cartilage of constitutively active human collagenase-3 (MMP-13) induces osteoarthritis in mice. J Clin Invest 2001; 107: 35-44.

[56] Little CB, Barai A, Burkhardt D, et al. Matrix metalloproteinase 13-deficient mice are resistant to osteoarthritic cartilage erosion but not chondrocyte hypertrophy or osteophyte development. Arthritis Rheum 2009; 60: 3723-33.

[57] $\mathrm{Hu} \mathrm{K}, \mathrm{Xu} \mathrm{L}, \mathrm{Cao} \mathrm{L}$, et al. Pathogenesis of osteoarthritis-like changes in the joints of mice deficient in type IX collagen. Arthritis Rheum 2006; 54: 2891-900.

[58] $\mathrm{Xu} \mathrm{L}$, Peng $\mathrm{H}$, Glasson S, et al. Increased expression of the collagen receptor discoidin domain receptor 2 in articular cartilage as a key event in the pathogenesis of osteoarthritis. Arthritis Rheum 2007; 56: 2663-73.

[59] van den Berg WB. Lessons from animal models of osteoarthritis. Curr Rheumatol Rep 2008; 10: 26-9. 